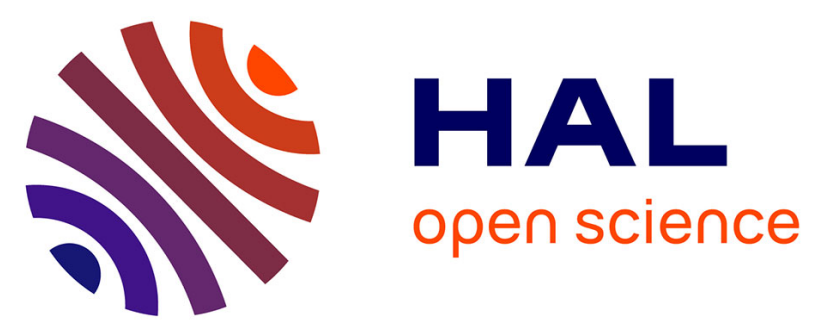

\title{
Etudes numérique et expérimentale des grandeurs mesurables en indentation sphérique: influence du frottement et du mode d'écrouissage du matériau
}

Olivier Bartier, Stanislaw Kucharski, Gerard Mauvoisin

\section{- To cite this version:}

Olivier Bartier, Stanislaw Kucharski, Gerard Mauvoisin. Etudes numérique et expérimentale des grandeurs mesurables en indentation sphérique: influence du frottement et du mode d'écrouissage du matériau. Matériaux \& Techniques, 2008, 96 (hors série), pp.59-70. 10.1051/mattech/2009016 . hal-00861798

HAL Id: hal-00861798

https://hal-univ-rennes1.archives-ouvertes.fr/hal-00861798

Submitted on 13 Sep 2013

HAL is a multi-disciplinary open access archive for the deposit and dissemination of scientific research documents, whether they are published or not. The documents may come from teaching and research institutions in France or abroad, or from public or private research centers.
L'archive ouverte pluridisciplinaire HAL, est destinée au dépôt et à la diffusion de documents scientifiques de niveau recherche, publiés ou non, émanant des établissements d'enseignement et de recherche français ou étrangers, des laboratoires publics ou privés. 


\title{
Etudes numérique et expérimentale des grandeurs mesurables en indentation sphérique: influence du frottement et du mode d'écrouissage du matériau Numerical and experimental study of spherical indentation data : influence of the friction coefficient and the hardening behaviour
}

\author{
O. Bartier ${ }^{1}$, S. Kucharski ${ }^{2}$ et G. Mauvoisin ${ }^{1}$ \\ ${ }^{1}$ : LARMAUR, Campus de Beaulieu, Université de Rennes1. 35042 RENNES \\ ${ }^{2}$ : Institute of Fundamental Technological Research of the Polish Academy of Science \\ Swietokrzyska 21,00-049 VARSOVIE, Poland \\ Olivier.bartier@univ-rennes1.fr
}

\begin{abstract}
Résumé - Les résultats d'indentation sphérique obtenus expérimentalement sur des aciers au carbone ayant subit un traitement de globulisation sont comparés à ceux obtenus numériquement. L'étude des courbes d'indentation montre que le frottement a une influence négligeable sur la courbe de chargement et faible sur la courbe de déchargement. Les simulations numériques montrent que le type d'écrouissage, isotrope ou cinématique, a une influence non négligeable sur la courbe de décharge. Le retour du matériau est beaucoup plus important dans le cas d'un écrouissage cinématique surtout à la fin de la décharge. L'étude de la morphologie des empreintes confirme ce résultat. La morphologie des empreintes obtenue numériquement montre aussi que la hauteur de bourrelet est influencée faiblement par le type d'écrouissage et fortement par le frottement. La hauteur du bourrelet diminue avec l'augmentation du coefficient de frottement. Cette étude montre que les calculs par éléments finis effectués en considérant une loi de comportement élasto-plastique isotrope, écrouissable avec un écrouissage cinématique non nul et un coefficient de frottement non nul entre le matériau et l'indenteur, permettent d'obtenir des résultats numériques d'indentation très proches de ceux obtenus expérimentalement.
\end{abstract}

Mots clefs : indentation sphérique, méthode des éléments finis, expérimentation, courbe d'indentation, empreinte

\begin{abstract}
The experimental results of spherical indentation obtained on globulized carbon steels are compared with those obtained numerically. The study of the indentation curves shows that friction has an negligible influence on the loading curve and a weak influence on the unloading curve. The numerical simulations show that isotropic or kinematic hardening has a considerable influence on the unloading curve. The unloading curves exhibit a greater curvature in the case of a kinematic hardening especially on the final part of theses curves. The study of the morphology of the imprints confirms this result. The imprint geometry obtained numerically shows that pile-up is slightly influenced by the type of hardening and strongly influenced by friction. The pile-up decreases with the increase in the friction coefficient. This study shows that finite elements simulations carried out by considering an isotropic elastic-plastic behavior, with kinematic hardening and a non-zero friction coefficient between the indenter and the indented material, make it possible to obtain numerical indentation results very close to those obtained in experiments.
\end{abstract}

Key-words : Spherical indentation, finite element method, experimentation, indentation curve, imprint geometry

\section{Introduction}

La caractérisation par indentation des matériaux consiste à rechercher les paramètres de la loi de comportement du matériau qui minimisent soit l'écart entre une courbe d'indentation expérimentale et un modèle issu de nombreuses simulations dans le cas de l'analyse indirecte, soit l'écart entre une courbe expérimentale et une courbe numérique dans le cas de l'analyse inverse.

Afin de caractériser un matériau par indentation, il faut s'assurer que les courbes expérimentales obtenues sur des «matériaux tests» se superposent aux courbes numériques obtenues par simulation de l'indentation avec les lois de comportement obtenues par traction sur les mêmes «matériaux tests ». Cette démarche de vérification, lorsqu'elle est suivie, montre généralement la nécessité de corriger les courbes expérimentales pour compenser le manque de rigidité du couple indenteur-banc d'essai.

Par ailleurs, pour une plus grande robustesse de la technique de caractérisation ou pour augmenter le nombre de paramètres identifiables par 
caractérisation il peut être judicieux de prendre en compte le profil de l'empreinte.

Pour une même loi de comportement, les courbes d'indentation numériques obtenues $\mathrm{F}(\mathrm{h})$ peuvent être différentes selon les conditions aux limites, les conditions de frottement au niveau du contact et le type d'écrouissage, isotrope ou cinématique du matériau étudié. L'influence de ces paramètres a déjà été souvent étudiée numériquement sur la courbe de chargement [1-6] et parfois sur la courbe de déchargement [4-6]. Quelques études numériques ont aussi porté sur l'influence de ces paramètres sur la géométrie de l'empreinte [4]. Cependant, à notre connaissance, peu d'études ont porté sur l'influence des conditions de frottement et d'écrouissage (isotrope ou cinématique) sur la cohérence entre les résultats expérimentaux et numériques.

Dans ce travail, la démarche de vérification de cohérence numérique-expérimentale a été suivie sur plusieurs aciers avec plusieurs types d'indenteur. Les courbes d'indentation expérimentales $\mathrm{F}(\mathrm{h})$ et les profils d'empreinte ont été comparés aux résultats numériques. L'influence du frottement et l'effet de l'écrouissage cinématique sur la courbe $\mathrm{F}(\mathrm{h})$ et sur la géométrie de l'empreinte sont analysés.

\section{Matériaux et méthodes}

\subsection{Microstructure et dureté}

Les essais expérimentaux de traction et d'indentation sont réalisés sur les quatre aciers présentés dans le tableau 1. Un traitement thermique ayant pour but l'obtention d'une microstructure globulisée a été effectué sur chaque matériau. La structure globulisée, plus homogène que la structure ferrito-perlitique initiale, doit permettre une meilleure reproductibilité des tests d'indentation. La figure 1 donne la micrographie de la structure de ces aciers globulisés après une attaque au nital. La dureté des matériaux étudiés est donnée dan le tableau 1.

Sur la figure 1 on observe que le traitement de globulisation n'a pas eu le même effet sur tous les matériaux. Sur la figure 1.c, représentant la microstructure de l'acier $\mathrm{C} 100$, on constate une distribution homogène de globules coalescés constitués de cémentite dans une matrice de ferrite.

\begin{tabular}{|c|c|}
\hline Désignation Matériaux & $\begin{array}{c}\text { Dureté Vickers } \\
\text { (30Kg) }\end{array}$ \\
\hline Acier C35 & 156 \\
\hline Acier C65 & 181 \\
\hline Acier C100 & 170 \\
\hline Acier 35CrMo4 & 198 \\
\hline
\end{tabular}

Tableau 1: Matériaux utilisés et leur dureté Table 1 : Materials and hardness

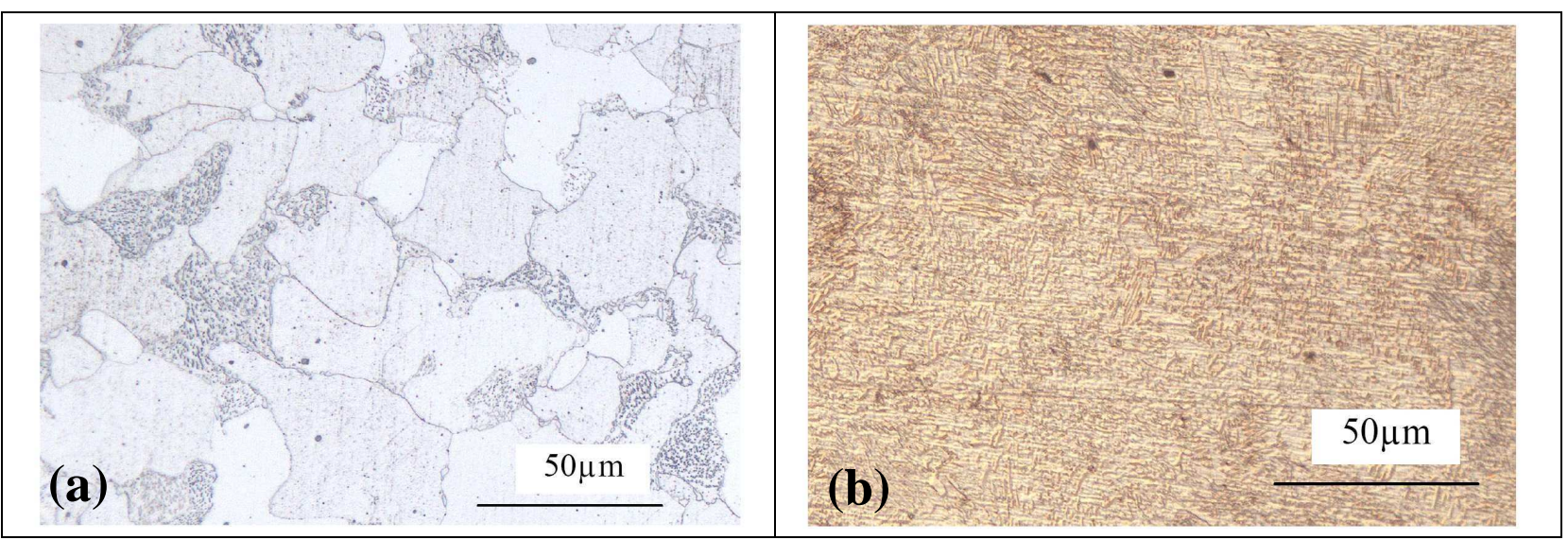




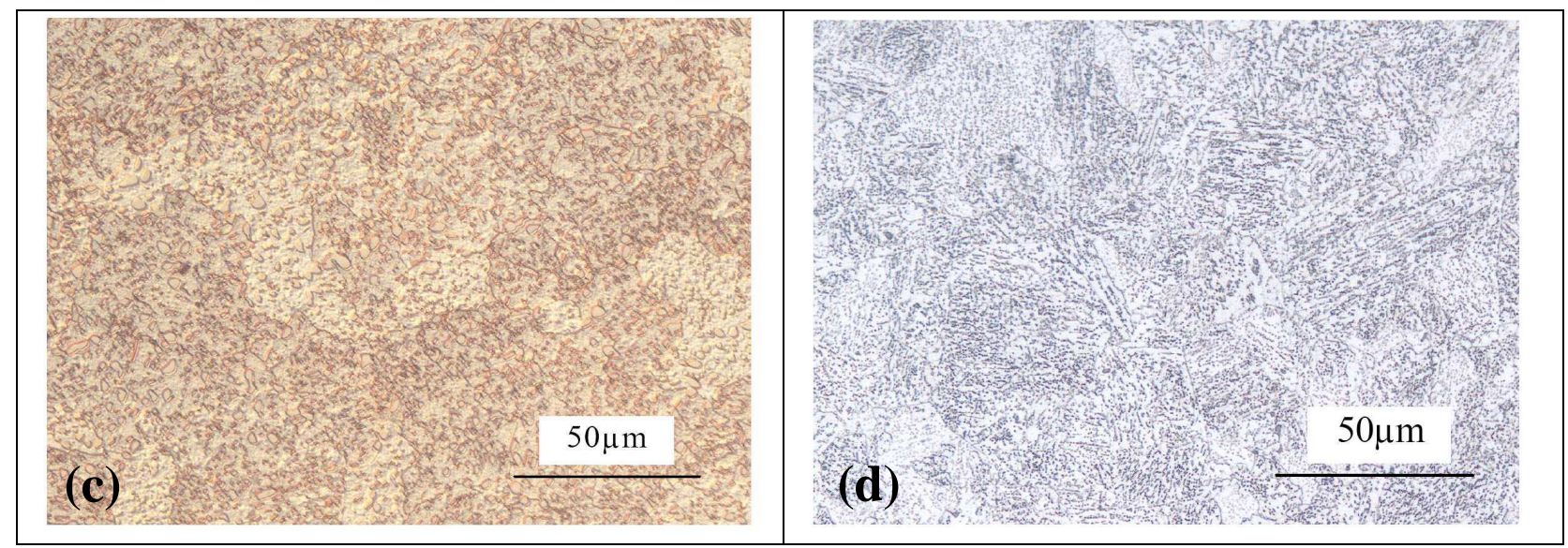

Figure 1 : Images métallographiques pour les matériaux étudiés, (a) C35 globulisé, (b) C65 globulisé, (c) C100 globulisé et (d) 35CrMo4 globulisé.

Figure 1 : Optical images after nital etching of the studied materials. Spheroidized steels : (a) AISI 1035, (b) AISI 1065, (c) AISI 1100 and (d) AISI 4135.

Le traitement thermique de globulisation de cet acier a été effectué, avant livraison, de manière optimale afin d'obtenir un matériau doux et ductile. Pour les autres aciers, C35, C65 et $35 \mathrm{CrMo} 4$, de structure initiale ferrito-perlitique, nous avons réalisé un traitement de globulisation, à $710^{\circ} \mathrm{C}$ durant $10 \mathrm{~h}$.

Après traitement de globulisation, on constate pour les matériaux $\mathrm{C} 65$ et $35 \mathrm{CrMo} 4$ la présence de quelques lamelles de perlite en plus de globules non coalescés (figure 1.b et 1.d). La globulisation partielle de ces matériaux n'a donc pas permis d'obtenir l'adoucissement optimal escompté. Pour l'acier C35, on distingue les grains de ferrite et les anciens grains de perlite dans lesquels les lamelles ont été remplacées partiellement par des globules. Ce cas de microstructure est intéressant car il conduit à une dispersion des courbes d'indentation dont on va discuter plus loin.

Les valeurs de dureté Vickers, données dans le tableau 1, confirment que l'adoucissement n'a pas été optimal pour les aciers C65 et $35 \mathrm{CrMo} 4$. On constate en effet que ces aciers sont plus durs que l'acier C100 alors qu'ils contiennent moins de carbone. On constate aussi que malgré le peu de carbone de l'acier C35, celui-ci n'a pas une dureté beaucoup plus basse que l'acier C100.

\subsection{Essais de traction}

Les essais de traction ont été effectués sur une machine pilotée en déplacement (type Lloyd LR $50 \mathrm{k}$ ) avec une vitesse d'avance de $2 \mathrm{~mm} / \mathrm{min}$. La courbe de traction est obtenue à partir d'éprouvettes cylindriques normalisées. L'allongement de la partie utile est mesuré à l'aide d'un capteur interférométrique de marque
Heidenhain permettent d'obtenir une précision de 0,05 microns sur les déplacements. La partie utile de l'éprouvette a un diamètre de $6 \mathrm{~mm}$ et une longueur effective de $50 \mathrm{~mm}$ (distance entre les couteaux du dispositif de mesure de déplacement).

Lors d'un essai de traction effectué sur une éprouvette ductile, la formation d'une striction se produit à partir d'une certaine valeur de déformation. Ce phénomène a pour effet une courbure marquée du profil de l'éprouvette dans le volume élémentaire représentatif où la contrainte et la déformation sont mesurées. Cette courbure du profil ajoute en plus des contraintes axiales, des contraintes de composante radiale, $\sigma_{r r}$ et circonférentielle $\sigma_{\theta \theta}$ positives. Il existe plusieurs méthodes afin de prendre en compte l'effet de la striction sur la relation contraintedéformation [7-11]. La plus ancienne est celle proposée par Bridgman [7]. Au contraire des autres méthodes, celle de Bridgman [7] a fait l'objet de nombreuses études numériques et expérimentales [10, 12-14]. De manière générale, les résultats de ces études montrent que cette technique permet de prendre en compte assez correctement l'influence de la striction sur la relation contrainte-déformation, surtout lorsque le rayon de courbure $\rho$ de l'éprouvette est grand devant la rayon $a$ de la section minimale [12-14]. Ceci explique que, malgré son imperfection, la méthode de Bridgman est la plus utilisée afin de déterminer la loi d'écrouissage post striction d'un matériau [12-16]. C'est cette méthode que nous utilisons ci-après.

Dans la méthode de Bridgman [7], il est nécessaire de déterminer, en plus de l'effort appliqué, le rayon $a$ de la section minimale ainsi que le rayon de courbure $\rho$ de la surface de l'échantillon au niveau de la zone de striction afin de parvenir à cette relation. Ces deux grandeurs 
ont été mesurés, à différents instants de l'essai de traction, à l'aide d'un système d'acquisition d'images. Avec ce système, la dérivation d'un polynôme représentant le profil de la surface au niveau de la striction a permis d'obtenir la valeur du rayon de courbure $\rho$. Les courbes rationnelles de traction obtenues à l'aide de la méthode de Bridgman [7] après striction sont représentées sur la figure 2 .

Le tableau 2 résume les valeurs des limites d'élasticité et des déformations à la rupture des différents échantillons testés.

\subsection{Simulations de l'indentation par éléments finis}

Les simulations par éléments finis du test d'indentation sont réalisées à l'aide du code de calcul Cast3M.

Les simulations numériques ont été effectuées avec les 2 types d'indenteur que nous utilisons en expérimentation. L'un des indenteurs est sphérique de rayon $0.5 \mathrm{~mm}$ et l'autre est un indenteur monobloc, axisymétrique mais non parfaitement sphérique. Pour les deux indenteurs nous avons considéré des propriétés élastiques correspondant à celles du carbure de Tungstène $(\mathrm{E}=600000 \mathrm{MPa}$ et $\mathrm{v}=0,28)$. La loi de comportement du matériau indenté est élastoplastique ( $\mathrm{E}=210000 \mathrm{MPa}$ et $\mathrm{v}=0,3)$, isotrope avec comme critère d'écoulement, celui de Von-Mises basé sur le second invariant du tenseur des contraintes $J_{2}(\bar{\sigma})$.

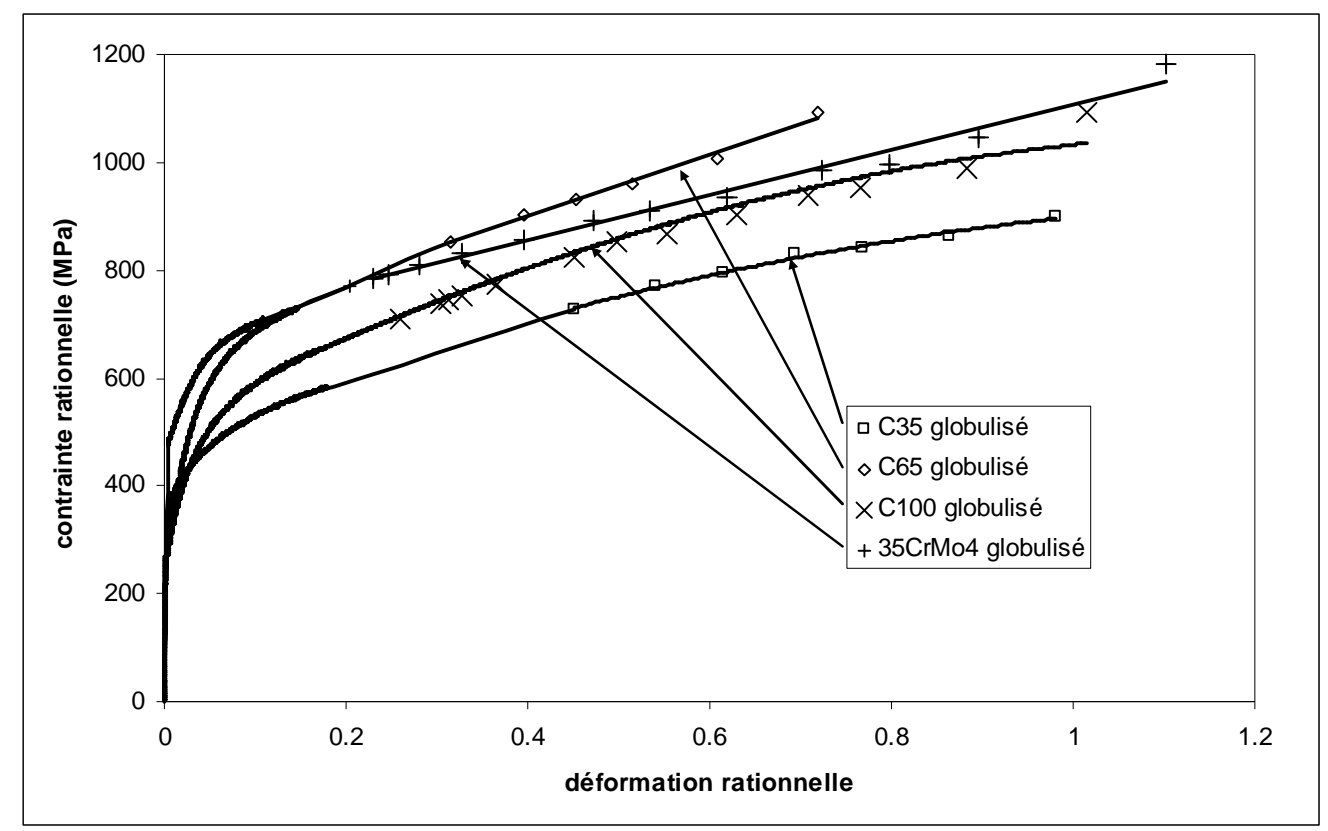

Figure 2: Evolution de la contrainte rationnelle en fonction de la déformation vraie obtenue avec la méthode de Bridgman.

\begin{tabular}{|l|c|c|}
\hline \multicolumn{1}{|c|}{ Matériau } & Limite d'élasticité (MPa) & A\% \\
\hline C35 globulisé & 300 & 28,6 \\
\hline C65 globulisé & 285 & 23,4 \\
\hline C100 globulisé & 195 & 30,8 \\
\hline 35CrMo4 globulisé & 472 & 21,8 \\
\hline
\end{tabular}

Tableau 2: Propriétés mécaniques obtenues à partir des courbes conventionnelles de traction.

Les simulations ont été effectuées pour un mode axisymétrique et pour les cas de contact avec et sans frottement ( $\mu=0,2$ et $\mu=0$ respectivement). Le modèle utilisé est celui présenté dans un précédent article [17].
Plusieurs types d'écrouissage ont été simulés. Le premier type d'écrouissage est un écrouissage isotrope avec évolution de l'écrouissage correspondant point par point à celui obtenu par essai de traction. C'est ce type d'écrouissage qui 
est utilisé de manière générale lors des simulations numériques. La surface de charge $f$ permettant de définir le seuil de plasticité dans le cas d'un matériau écrouissable isotrope s'écrit dans ce cas :

$$
f=J(\bar{\sigma})-\left(\sigma_{y}+R\right)
$$

Avec $\sigma_{\mathrm{y}}$ la limite d'élasticité du matériau et $\mathrm{R}$ la variable scalaire caractérisant l'augmentation de la limite d'élasticité lors de l'écrouissage isotrope.

Le deuxième type d'écrouissage est un écrouissage purement cinématique, pour lequel la surface de charge $f$ s'écrit :

$$
f=J(\overline{\bar{\sigma}}-\overline{\bar{X}})-\sigma_{y}
$$

Où $\stackrel{\bar{X}}{X}$ est la variable d'écrouissage de nature tensorielle gouvernant la translation de la surface de charge et donc la position du nouveau centre du domaine élastique.

La loi d'écrouissage cinématique non linéaire utilisée lors des simulations est celle d'Armstrong -Frederick qui s'écrit traditionnellement dans le cas d'une traction simple sous la forme suivante :

$$
\sigma=\sigma_{y}+\frac{C}{\gamma}\left(1-e^{-\gamma \varepsilon} p\right)
$$

où $\mathrm{C}$ et $\gamma$ caractérisent respectivement la vitesse de translation de la surface de charge et la vitesse de saturation du durcissement cinématique au cours de la déformation plastique.

L'avantage de la loi d'écrouissage cinématique présentée ci-dessus (équation 3) est qu'elle est implémentée dans le logiciel par éléments finis Cast3M. L'inconvénient est qu'elle ne suit pas fidèlement l'évolution de l'écrouissage obtenue par traction (figure 2). Il est donc difficile d'examiner l'influence du type d'écrouissage en comparant les résultats obtenus avec la loi d'écrouissage cinématique d'Armstrong-Frederick et ceux obtenus avec la loi d'écrouissage isotrope obtenu par essai de traction.
En conséquence, pour comparer numériquement l'influence du type d'écrouissage, isotrope ou cinématique, sur les résultats d'indentation, la loi d'écrouissage isotrope de Voce a été choisie. Cette lois 'exprime de manière analogue à l'équation 3 :

$$
\sigma=\sigma_{y}+Q\left(1-e^{-b \varepsilon_{p}}\right)
$$

Où $\varepsilon_{\mathrm{p}}$ est la déformation plastique cumulée et $Q$ et $b$ sont des constantes qui ont la même influence sur l'écrouissage isotrope du matériau que les paramètres $C$ et $\gamma$.

Les valeurs des paramètres des lois d'écrouissage d'Armstrong-Frederick et de Voce obtenues en se calant sur les résultats obtenus par traction sont données dans le tableau 4.

\subsection{Test d'indentation sur banc expérimental}

Les tests d'indentation ont été réalisés sur un banc d'indentation instrumenté conçu au sein de notre laboratoire. L'effort maxi appliqué sur l'indenteur est de $200 \mathrm{~N}$. Pour nos essais, la vitesse de déplacement est de $2 \mu \mathrm{m} / \mathrm{s}$.

Nos essais d'indentation à chargement croissant ont été effectués avec deux types d'indenteur. Le premier indenteur, appelé par la suite monobloc, est en carbure de tungstène taillé dans la masse (figure 3a, et 3b). La partie utile de l'indenteur n'est pas parfaitement sphérique. Le rayon équivalent de l'extrémité est plus faible que celui souhaité, de $0,5 \mathrm{~mm}$. Le deuxième indenteur, acheté dans le commerce, est constitué d'une bille en carbure de tungstène de diamètre $\mathrm{D}=1 \mathrm{~mm}$ sertie dans un porte indenteur en acier (figure 3.c).

Afin de s'affranchir des déformations entre la bille et son support dans le cas de l'indenteur sphérique du commerce, une correction des courbes d'indentation, basée sur le calcul de compliance selon la méthode d'Oliver and Pharr [18], a été réalisée.

\begin{tabular}{|c|c|c|c|c|}
\hline & C35 & C65 & C100 & 35 CrMo4 \\
\hline$\gamma$ ou b & 30 & 25,6 & 20,4 & 20,9 \\
\hline Q ou C/ $\gamma(\mathrm{MPa})$ & 256 & 443 & 482 & 291 \\
\hline
\end{tabular}

Tableau 4 : Valeurs des paramètres des lois d'écrouissage d'Armstrong-Frederick et de Voce 

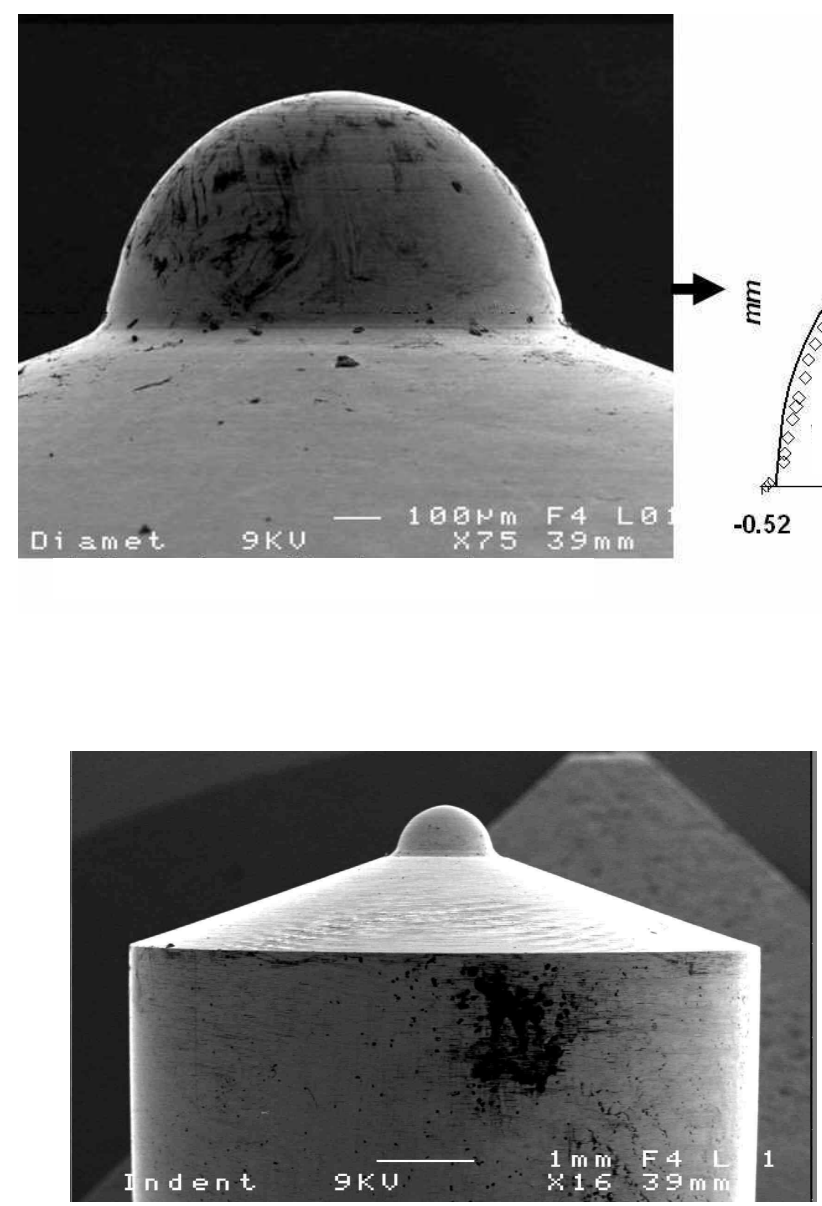

(b)

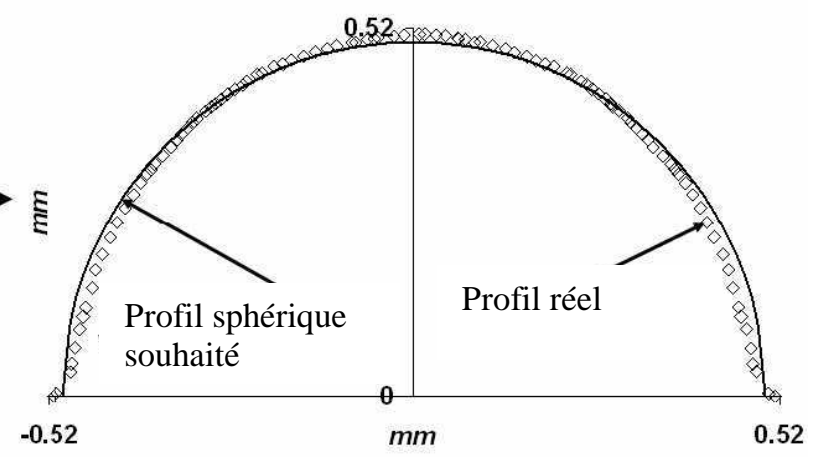

(a)

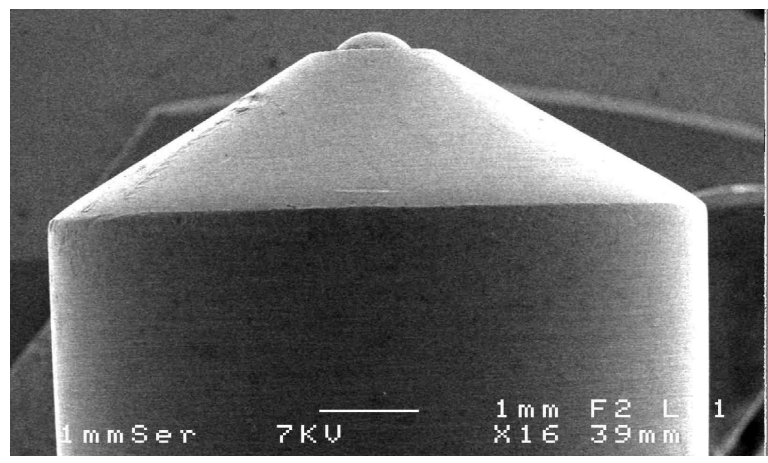

(c)

Figure 3 : Photos obtenues par MEB .

(a) et (b) : indenteur monobloc

(c) : indenteur avec bille sertie

\section{Résultats}

Sont présentées ici les courbes d'indentation obtenues sur les 4 matériaux d'étude ainsi que les profils des empreintes mesurés par profilomètre (T8000 nanoscan, Hommel Etamic) et par microscope confocale. Les mesures sont comparées aux valeurs numériques obtenues par la méthode des éléments finis dans le cas où les matériaux testés possèdent une loi de comportement isotrope à écrouissage isotrope avec comme lois d'écrouissage, celles présentées à la figure 2 .

La figure 4 présente les courbes d'indentation expérimentales, après correction de rigidité, et les courbes numériques obtenues pour les 2 indenteurs utilisés.
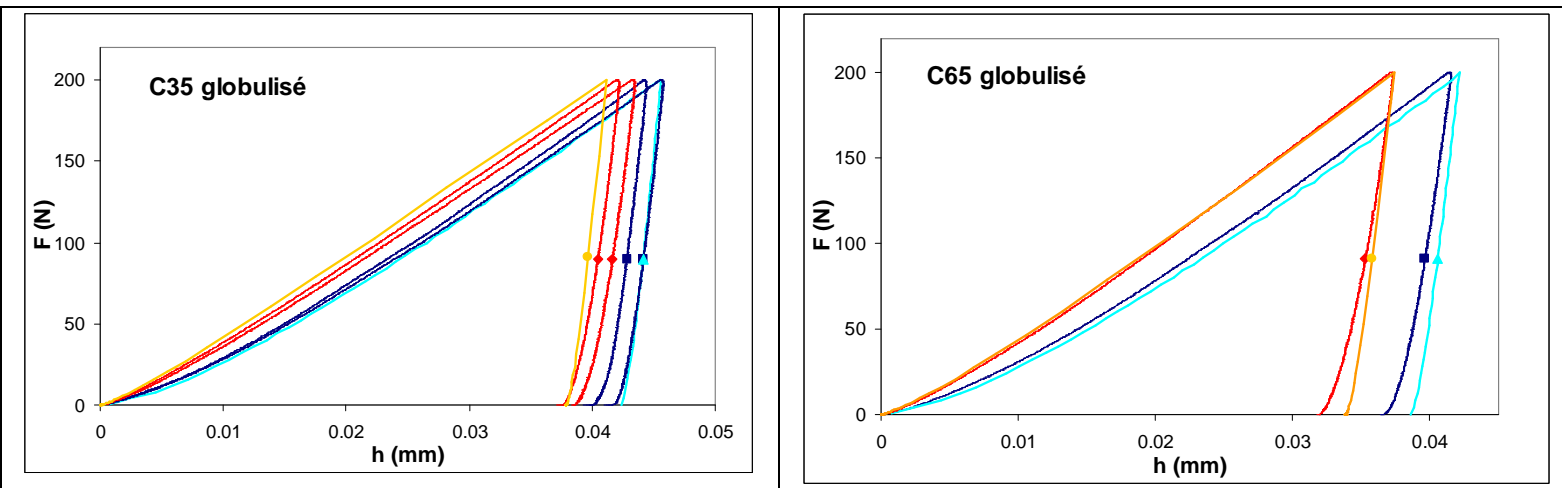

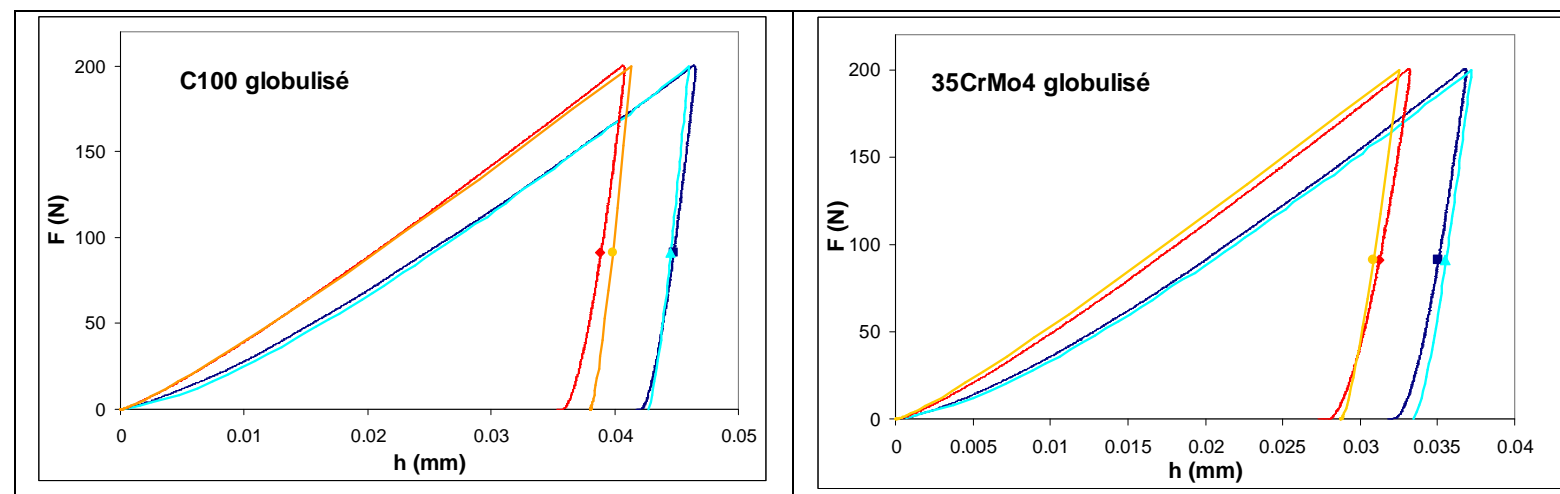

Figure 4 : Comparaison entre les résultats numériques et expérimentaux obtenus avec l'indenteur monobloc et la bille sertie. résultats expérimentaux : - $\mathbf{\square}$ indenteur monobloc, $-\varangle$ indenteur avec bille sertie ; résultats numériques obtenus avec écrouissage isotrope et coefficient de frottement nul :- - indenteur monobloc, —— indenteur avec bille sertie.

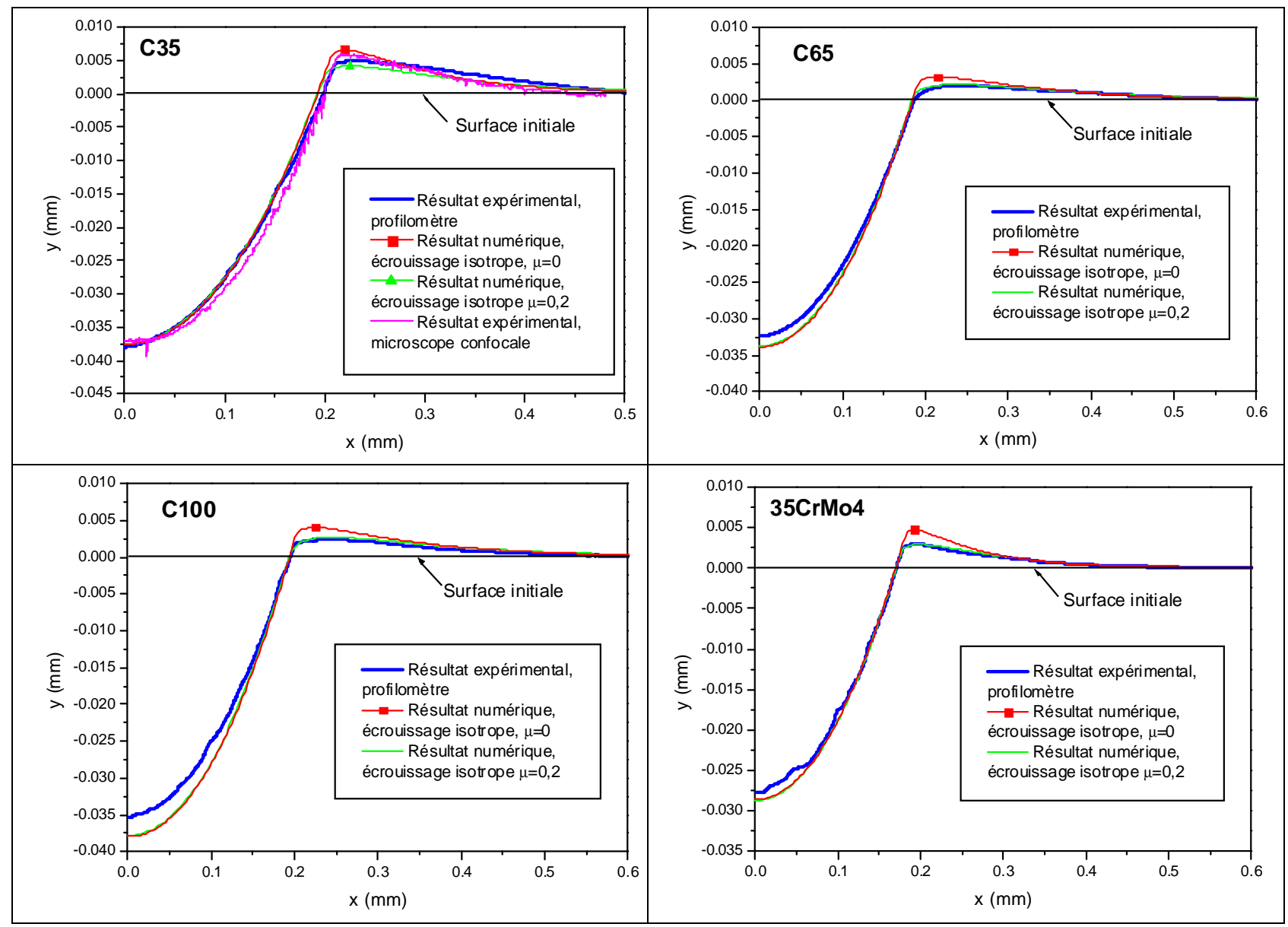

Figure 5 : Profils d'empreintes d'indentation, avec indenteur à bille sertie, déterminés numériquement et expérimentalement par profilomètre

La figure 5 montre les profils des empreintes d'indentation obtenues expérimentalement et numériquement avec l'indenteur à bille sertie. Pour tous les aciers étudiés, les empreintes ont été obtenues avec le profilomètre. Afin de confirmer les résultats obtenus par profilométrie, les empreintes ont aussi été analysées par microscopie confocale dans le cas de l'acier C35. Une comparaison des résultats obtenus par les deux méthodes est présentée sur la figure 5 dans le cas de l'acier C35. Les 
simulations numériques ont été effectuées avec et sans frottement ( $\mu=0$ et $\mu=0.2$ respectivement).

\section{Discussion}

Les résultats présentés ci-dessus méritent de nombreux commentaires que l'on donne ciaprès en distinguant les résultats obtenus sur les courbes de chargement et les résultats obtenus sur les profils d'empreinte.

\subsection{Courbes F(h)}

\subsubsection{Reproductibilité}

Pour les aciers C65, C100 et 35CrMo4, une seule courbe expérimentale est donnée à chaque fois sur la figure 4 car la reproductibilité des résultats est excellente.

En revanche, pour l'acier C35 nous avons représenté plusieurs courbes expérimentales sur la figure 4-a afin de montrer le manque de reproductibilité. Nous expliquons ceci par l'hétérogénéité de la microstructure de cet acier, visible sur la figure 1-c. En effet, le matériau C35 étant ferrito perlitique avant le traitement de globulisation, les globules de cémentite se situent dans les anciens grains de perlite. Ainsi, la répartition non homogène des globules de cémentite dans le matériau s'apparente à une hétérogénéité du matériau à l'échelle de l'indentation, notamment dans les premiers instants du test. Ce phénomène, qui n'a pas été rencontré sur les autres matériaux, montre à quel point le test d'indentation est sensible à l'uniformité de la microstructure en regard de l'échelle d'étude et nous conforte dans notre choix d'étudier les matériaux métalliques sous forte charge alors que la tendance générale privilégie les micro voire les nano charges.

4.1.2. Différence entre indenteur à bille sertie et indenteur monobloc

La figure 4 montre que pour les 4 matériaux, la pénétration de l'indenteur monobloc, à même effort appliqué, est plus élevée que celle de l'indenteur à bille sertie. Ce résultat est du au fait que le rayon de l'indenteur monobloc est globalement plus faible que celui de l'indenteur avec bille sertie (figure 3.a).

\subsubsection{Correspondance de la courbe de chargement}

Sur la figure 4, on observe que globalement les courbes numériques et expérimentales se superposent assez bien au niveau du chargement. Ceci montre que la loi de comportement déterminée par essai de traction et utilisée dans les codes de calculs par éléments finis permet d'obtenir correctement la réponse à l'indentation des aciers testés.

\subsubsection{Correspondance de la courbe de déchargement}

Sur la figure 4, on observe que les courbes numériques et expérimentales ne se superposent pas au niveau du déchargement. Deux raisons peuvent expliquer la différence entre les décharges numériques et les décharges expérimentales.

La première raison concerne l'influence du frottement entre l'indenteur et le matériau. Nous avons réalisé des simulations de l'indentation sur les matériaux $\mathrm{C} 65$ et $32 \mathrm{CrMo} 4$ avec deux coefficients de frottement $\mu=0$ et $\mu=0,2$. La figure 6 montre que le frottement n'a pas d'influence sur le chargement et trop peu d'influence sur la décharge pour expliquer la différence constatée entre les courbes de décharge numérique et expérimentale. D'une manière générale, les courbes d'indentation obtenues pour les matériaux testés montrent que l'augmentation du coefficient de frottement n'accentue que très légèrement le retour du matériau lors de l'indentation. Ces résultats sont cohérents avec ceux obtenus par d'autres chercheurs dans le cas de l'indentation sphérique [1-3].

$\mathrm{La}$ seconde raison est le type d'écrouissage. Sur la courbe de déchargement, on constate que quelque soit le matériau testé et l'indenteur utilisé, le retour élastique mesuré expérimentalement est toujours plus important que celui calculé numériquement. Ce phénomène a déjà été observé par ailleurs $[4,6,19]$ et a été attribué à une plastification du matériau indenté non prise en compte par le modèle numérique lorsque l'écrouissage isotrope est utilisé $[4,6]$. Les courbes d'indentation numériques représentées sur les graphes de la figure 4 ont été obtenues avec un écrouissage isotrope. La figure 7 montre que lorsque l'écrouissage du matériau est cinématique (loi d'Armstrong-Frederick), le retour du matériau lors de la décharge est plus élevé que celui obtenu lorsque l'écrouissage du matériau est isotrope (loi de Voce). On remarque sur cette figure que l'écrouissage cinématique accentue surtout le retour du matériau en fin de décharge. Ce résultat est en accord avec ceux obtenus numériquement par Pane et Blank [4] et Hubert et Tsakmakis [6] dans le cas de l'indentation sphérique de matériaux élastoplastiques avec écrouissage cinématique. La 
figure 7 montre toutefois que la loi d'écrouissage cinématique pure considérée ici ne permet pas d'obtenir la correspondance attendue entre les courbes numériques et expérimentales de la décharge. On a constaté, sur des matériaux similaires, qu'une bonne correspondance ne pouvait être obtenue qu'en considérant une loi d'écrouissage mixte associant écrouissage isotrope et cinématique à plusieurs termes [20].

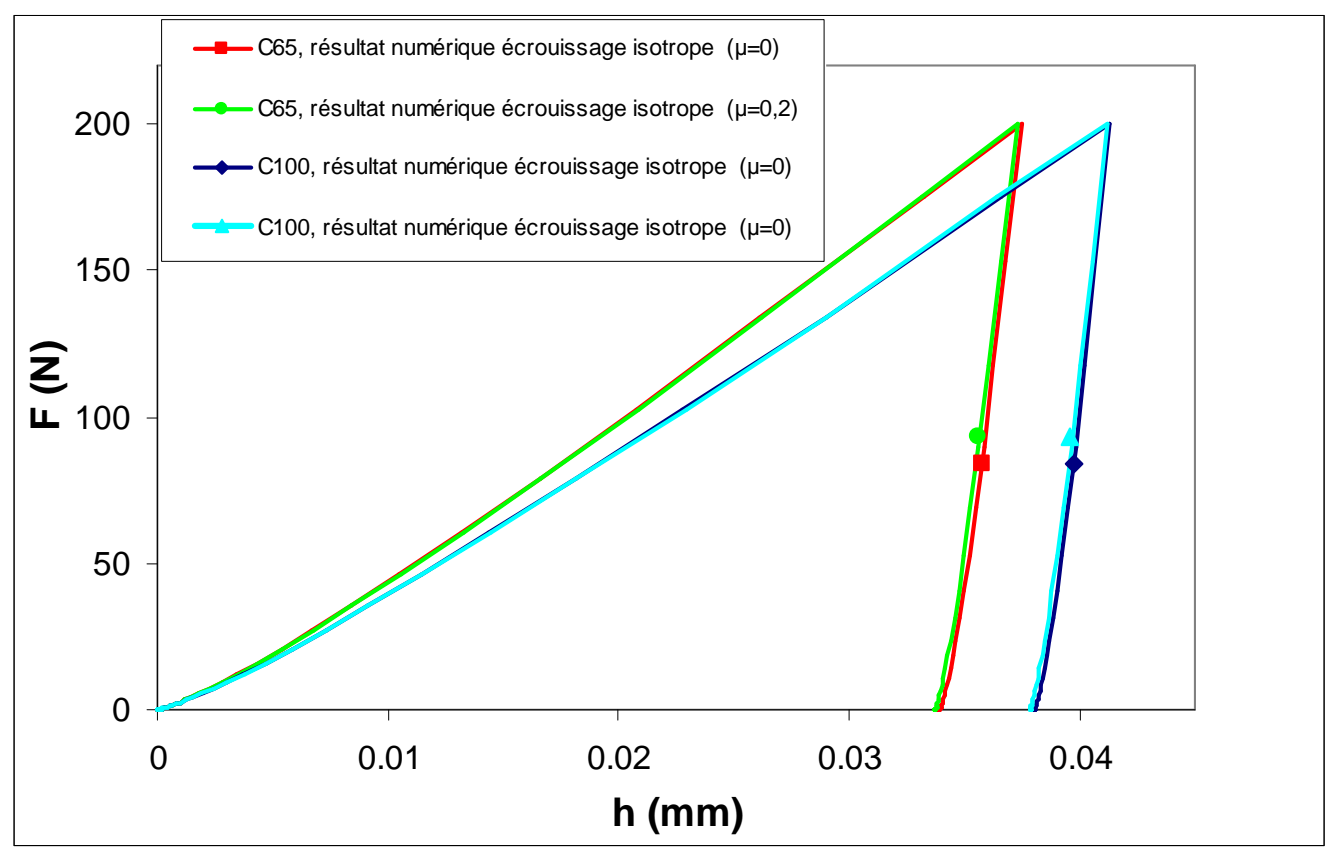

Figure 6 : influence du coefficient de frottement sur la courbe d'indentation.

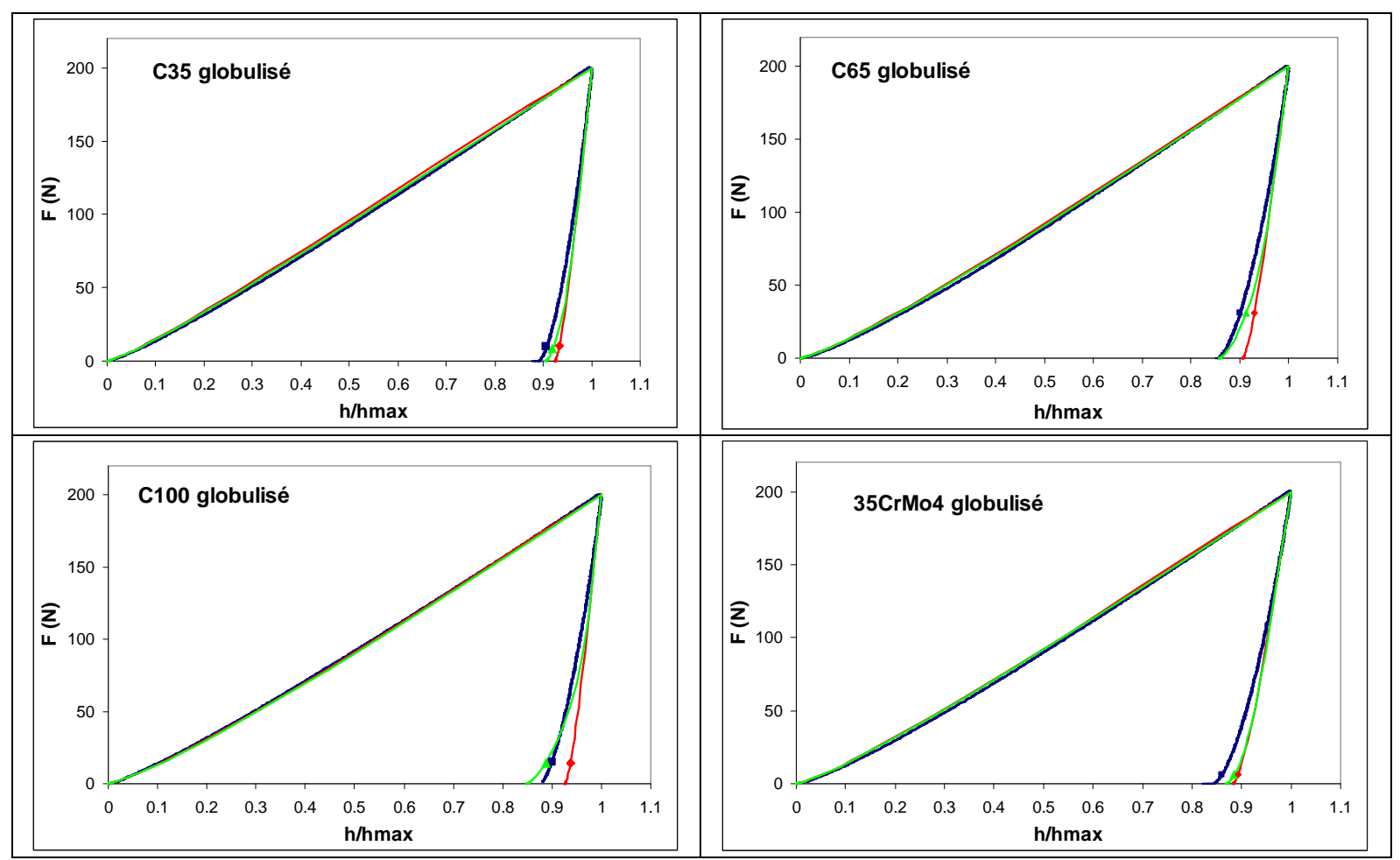

Figure 7 : Influence du type d écrouissage sur la courbe de décharge. Résultat expérimental : 一 - ; résultats numériques :- - écrouissage isotrope, loi de Voce ; - - - écrouissage cinématique, loi d'Armstrong-Fredérik. 


\subsection{Profil des empreintes}

\subsubsection{Influence du frottement}

La figure 5 montre que, globalement, les empreintes obtenues numériquement se superposent à celles obtenues expérimentalement. Cependant, la hauteur de bourrelet obtenue numériquement est généralement surestimée par rapport à celle obtenue expérimentalement. On rappelle qu'une dispersion des résultats expérimentaux a été obtenue dans le cas de l'acier C35 (figure 4). Pour cet acier, l'empreinte expérimentale présentée figure 5 est la plus proche de celle obtenue numériquement.

La figure 5 montre que le coefficient de frottement n'a que très peu d'influence sur la profondeur de l'empreinte. En revanche, il a une influence significative sur la hauteur du bourrelet et donc sur le rayon de contact entre l'indenteur et le matériau indenté. La hauteur de bourrelet est plus faible lorsque le coefficient de frottement est élevé. Ce résultat est cohérent avec celui trouvé numériquement par Taljat et Pharr [21] dans le cas de matériaux élastiques plastiques peu écrouissables. L'utilisation d'un coefficient de frottement non nul dans les simulations numériques a pour conséquence l'obtention de hauteurs de bourrelet plus proches de celles obtenues expérimentalement, sauf pour l'acier C35 pour lequel on a noté une dispersion des résultats.

\subsubsection{Influence du durcissement par écrouissage}

On constate sur la figure 5 que les hauteurs de bourrelet obtenues pour les aciers C35 et $35 \mathrm{CrMo} 4$ sont plus élevées que celles obtenues pour les autres aciers. Il a été montré dans de précédents articles que la hauteur du bourrelet dépendait significativement de l'exposant d'écrouissage du matériau [17,21]. Plus l'écrouissage du matériau est faible, plus la hauteur du bourrelet est importante. La figure 1 et les valeurs des paramètres $\mathrm{C} / \gamma$ et $\mathrm{Q}$ données dans le tableau 1 montrent que les aciers C35 et $35 \mathrm{CrMo} 4$ sont les matériaux les moins écrouissables parmi ceux étudiés. Enfin, sur la figure 5 on observe que la hauteur du bourrelet obtenue pour l'acier C35 est plus élevée que celle obtenue pour l'acier $35 \mathrm{CrMo}$. Ce résultat est du à la différence de limite d'élasticité entre les aciers C35 et 35CrMo4. En effet, dans de récents articles [17, 21], il a été montré que la hauteur du bourrelet dépendait du rapport entre les taux de déformation élastique et plastique obtenus dans le matériau indenté pour un effort d'indentation donné. Plus le rapport E/ $\sigma_{\mathrm{y}}$ du matériau indenté est élevé plus le taux de déformation plastique dans le matériau indenté est élevé et donc plus la hauteur du bourrelet est élevée. Dans notre cas, la plus grande valeur de la limite d'élasticité de l'acier $35 \mathrm{CrMo} 4$ a donc pour conséquence un taux de déformation plastique plus faible lors de l'indentation et donc une hauteur de bourrelet plus faible.

\subsubsection{Influence du type d'écrouissage}

Les profils d'empreintes obtenus par simulation de l'indentation en considérant un écrouissage cinématique non linéaire de type ArmstrongFrederick sont représentés sur les graphes de la figure 8. Ils sont comparés à ceux obtenus en considérant un écrouissage isotrope non linéaire de type Voce afin d'observer l'influence du type d'écrouissage sur la morphologie de l'empreinte. La figure 8 confirme que l'utilisation d'un écrouissage cinématique dans les simulations numériques a pour conséquence la diminution de la valeur de la profondeur résiduelle après décharge. Cette figure montre aussi que la prise en compte d'un écrouissage cinématique plutôt qu'un écrouissage isotrope permet de diminuer la différence entre les résultats numériques et expérimentaux. On observe enfin sur la figure 8 que le type d'écrouissage, isotrope ou cinématique, n'a par contre que très peu d'influence sur la hauteur du bourrelet

\section{Conclusion}

L'étude a consisté à vérifier la cohérence entre les résultats numériques et les résultats expérimentaux, en ce qui concerne les courbes d'indentation $\mathrm{F}(\mathrm{h})$ et les profils d'empreinte, sur plusieurs aciers avec plusieurs types d'indenteur.

L'étude à d'abord montré que le matériau devait avoir une structure suffisamment fine et homogène, à l'échelle de l'indentation, pour obtenir des courbes reproductibles utilisables dans une démarche de caractérisation des matériaux.

Le frottement a une influence plus négligeable sur la courbe de chargement que sur la courbe de déchargement. Il a par ailleurs une influence significative sur la hauteur du bourrelet. $\mathrm{La}$ hauteur $\mathrm{du}$ bourrelet diminue avec l'augmentation du coefficient de frottement. En conséquence, les simulations numériques doivent être réalisées avec le coefficient de frottement le plus réaliste possible dans le cas où la géométrie 


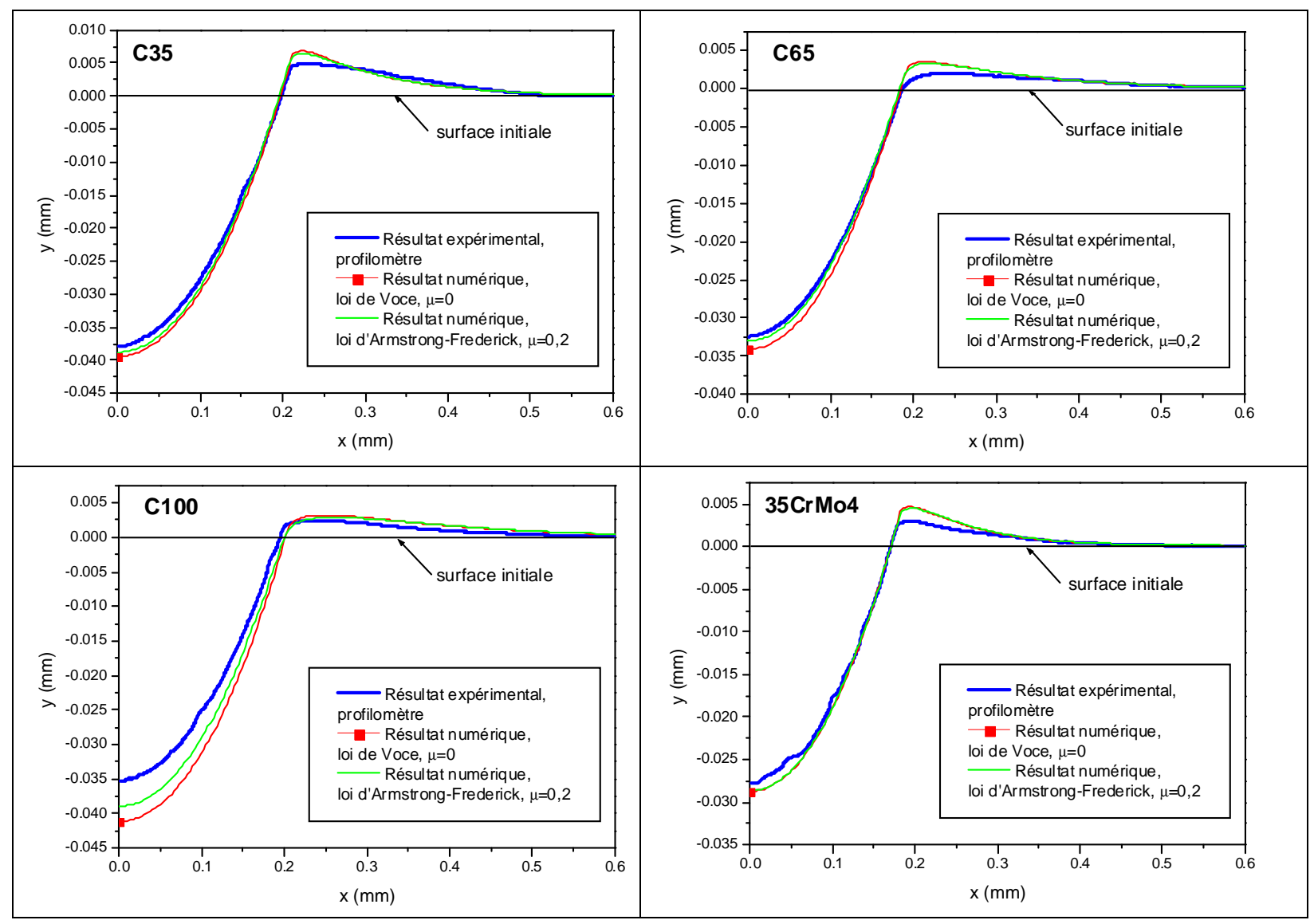

Figure 8 : Influence du type d'écrouissage sur le profil des empreintes d'indentation obtenus numériquement dans le cas de l'indenteur à bille sertie

Pour notre étude, les résultats expérimentaux sont plus proches de ceux obtenus numériquement lorsque l'on considère un frottement non nul entre l'indenteur et le matériau indenté.

Le type d'écrouissage, isotrope ou cinématique, a une grande influence sur la courbe de décharge. La remontée de matière sous l'indenteur durant la décharge est beaucoup plus importante dans le cas d'un écrouissage cinématique surtout à la fin de la décharge. Les études numériques et expérimentales de la morphologie de l'empreinte confirment ce résultat. Par ailleurs, l'étude de la morphologie de l'empreinte montre que le type d'écrouissage n'a en revanche que très peu d'influence sur la hauteur de bourrelet. Dans le cas des aciers au carbone présentés dans cet article, la prise en compte d'un frottement non nul associé à un écrouissage cinématique permet de mieux faire coïncider les résultats numériques à ceux obtenus expérimentalement.

En conclusion, le test d'indentation peut être un outil intéressant afin d'approcher la loi de comportement de matériaux sans réaliser d'essai de traction, ce qui est particulièrement intéressant lorsqu'on ne dispose que d'un petit volume de matière. Dans une démarche de caractérisation mécanique de matériaux, la géométrie de l'empreinte peut être ajoutée au champ de données expérimentales. Cependant, en l'absence de quelques précautions, les différences observées entre les résultats expérimentaux et les résultats numériques, tant sur les courbes de chargementdéchargement que sur le profil des empreintes, montrent que le problème d'identification de propriétés mécaniques par indentation n'est pas trivial.

\section{Remerciements}

Ce travail a été mené dans le cadre d'un Programme Hubert Curien «Polonium » financé conjointement par le Ministère des Affaires étrangères, avec le soutien du Ministère de l'Education nationale, de l'Enseignement supérieur et de la Recherche pour la partie française. 


\section{Bibliographie}

[1] C. Zabolestani Moradi. Development of a methodology to determine the flow stress from instrumented indentation testing using inverse analysis. Thesis, The Ohio State University, 2004 [2] A. Nayebi, R. El Abdi, O. Bartier, G. Mauvoisin, Mech. Mat. 34 (2002) 243-254

[3] T. Seifert, Identification of Material Parameters Using Instrumented Indentation Test Data, Thèse, University of Stuttgart, 2003

[4] I. Pane, E. Blank, Int. J. Solids Struct. 43 (2006) 2014-2036

[5] N. Huber, CH. Tsakmakis, J. Eng. Mat. Tech. 120 (1998) 143-148

[6] N. Huber, Ch. Tsakmakis, Mech. Mat. 27

(1998) 241-248

[7] P. Bridgman, Trans. Am. Soc. Met. 32 (1944)

553-574

[8] N. Davidenkov, N. Spiridonova, Proc. Amer.

Soc. Test. Mat. 46 (1946) 1147-1158

[9] L. Dietrich, J. Miastkowski, W. Szczepiński:

Nośność graniczna el ementów konstrukcji.

Warszawa, PWN, (1970). Cité par Gromada [10].

[10] M. Gromada, G. Mishuris, A. Öchsner.

Critical Analysis of the Evaluation of Plastic

Material Properties Obtained from Standard

Round Tensile Specimens. In: Proceedings of the

Third International Conference on Mathematical

Modeling and Computer Simulation of Materials

Technology (MMT-2004), Ariel, Israel, 2004,

pp.78-87

[11] G. Mirone, Int. J. Solids Struct 41 (2004)

3545-3564.

[12] E. E. Cabezas, D. J. Celentano, Finite

Elements Anal. Des. 40 (2004) 555-575

[13] D. J Celentano, E. E Cabezas, C. M Garcia

and A. E Monsalve. Model. Simulation Mat. Sci.

Eng. 12 (2004) S425-S444

[14] C. García-Garino, F. Gabaldón, J. M.

Goicolea, Finite Elements Anal. Des. 42 (2006)

$1187-1197$

[15] Y. Ling. AMP J. Tech. 5 (1996) 37-48

[16] Y.Tillier, Identification par analyse inverse du comportement mécanique des polymères

solides, applications aux sollicitations

multiaxiales et rapides, Thèse, Ecole Nationale

Supérieure des Mines de Paris, 1998

[17] X. Hernot, O. Bartier, Y. Bekouche, G. Mauvoisin, R. El Abdi, Int. J. Solids Struct. 43 (2006) 4136-4153

[18] W.C. Oliver, G.M. Pharr, J. Mater. Res. 7 (1992) 1564-1583

[19] D. J. Strange, A. K. Varshneya. J. Mat. Sci. 36 (2001) 1943 - 1949.
[20] J.-M. Collin, T. Parenteau, G. Mauvoisin and P. Pilvin, Comput .Mat. Sci. (2009) doi :10.1016/j.commatsci.2009.03.016

[21] B. Taljat, G.M. Pharr, Int. J. Solids Struct. 41 (2004) 3891-3904. 